\title{
Label-free optical spectroscopy for characterizing binding properties of highly sensitive nanocrystalline cellulose-graphene oxide based nanocomposite towards nickel ion
}

\begin{abstract}
Surface plasmon resonance (SPR) is a label-free optical spectroscopy that is widely used for biomolecular interaction analysis. In this work, SPR was used to characterize the binding properties of highly sensitive nanocrystalline cellulose-graphene oxide based nanocomposite (CTA-NCC/GO) towards nickel ion. The formation of CTA-NCC/GO nanocomposite has been confirmed by FT-IR. The SPR analysis result shows that the CTA-NCC/GO has high binding affinity towards $\mathrm{Ni}^{2+}$ from 0.01 until $0.1 \mathrm{ppm}$ with binding affinity constant of $1.620 \times 10^{3} \mathrm{M}^{-}$ 1. The sensitivity for the CTA-NCC/GO calculated was $1.509^{\circ} \mathrm{ppm}^{-1}$. The full width at half maximum (FWHM), data accuracy (DA), and signal-to-noise ratio (SNR) have also been determined using the obtained SPR curve. For the FWHM, the value was $2.25^{\circ}$ at 0.01 until $0.08 \mathrm{ppm}$ and decreases to $2.12^{\circ}$ at 0.1 until $10 \mathrm{ppm}$. The DA for the SPR curves is the highest at 0.01 until $0.08 \mathrm{ppm}$ and lowest at 0.1 until $10 \mathrm{ppm}$. The SNR curves mirrors the curves of SPR angle shift where the SNR increases with the $\mathrm{Ni}^{2+}$ concentrations. For the selectivity test, the CTA-NCC/GO has the abilities to differentiate $\mathrm{Ni}^{2+}$ in the mixture of metal ions.
\end{abstract}

Keyword: Graphene oxide; Label-free; Nanocrystalline cellulose; Nickel ion; Sensing; Surface plasmon resonance. 